\title{
A Clinical Study of Efficacy of Hydrocortisone Compared With Hyaluronic Acid After Arthrocentesis In TMJ Disorders
}

Pavan Kumar B ${ }^{1}$, Haripriya Chari², Mohan AP³, Brahmaji Rao J ${ }^{4}$

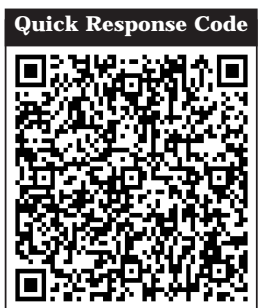

doi: $10.5866 / 2016.8 .10141$

${ }^{1}$ Professor and Head of the Department

2Senior Lecturer

${ }^{3}$ Professor

${ }^{4}$ Professor

Department of Oral and Maxillofacial Surgery

Kamineni Institute of Dental Sciences

Narketpally, Nalgonda

\section{Article Info:}

Received: J uly 11, 2016

Review Completed: August 10, 2016

Accepted: September 9, 2016

Available Online: September, 2016 (www.nacd.in)

(C) NAD, 2016 - All rights reserved

Email for correspondence:

pavankumarbatchu40@gmail.com

\begin{abstract}
:
Temporo-mandibular J oint disorders can be treated with varying methods ranging from conservative management, including drug therapy and occlusal splint therapy, to surgical management which should ideally be the last resort. After the introduction of arthrocentesis as a non-invasive and conservative treatment modality, it was observed that many cases did not require the surgery of the temporomandibular joint.

In the present study the efficacy of intra-articular injections of hyaluronic acid and hydrocortisone were compared which were given after temporomandibular joint arthrocentesis. Both drugs were able to demonstrate a reduction of pain and improvement in mouth opening, reducing joint sounds. We noticed that the efficacy of intra-articular injection of hyaluronic acid is better compared to intra-articular injection of hydrocortisone.

Hence, we conclude that arthrocentesis foll owed by intra-articular injection of hyaluronic acid is better compared to intra-articular injection of hydrocortisoneand is a simpleand non-invasive method for temporomandibular joint disorders such as anterior disc displacement with and without reduction. Finally it presents a low incidence of intraoperative and postoperative complications and however additional research may require for long term evaluation of the results.
\end{abstract}

Key words: Arthrocentesis, Hyaluronic acid, Hydrocortisone, Clicking sounds.

\section{INTRODUCTION}

Temporo-mandibular disorders (TMD) are a heterogeneous group of pathologies affecting the temporomandibular joints (TMJ ), the masticatory muscles or both. The disorders are characterized by a classically described triad of clinical signs: muscle and/or TMJ pain, TMJ sounds and restriction, deviation or deflection of mouth opening path. The objective of management for any disease process is the full restoration of function with improvement of the quality and quantity of life. Many noninvasive approaches have been developed to alleviate the pain and functional complaints of patients suffering from temporomandibular disorders among which are occlusal splint therapy, physiotherapy, complimentary medicine, pharmacotherapy and 
occlusal treatments. Recent reports have pointed out the importance of joint lubrication for a correct joint function, also hypothesizing that abnormalities of the joint lubrication system may play a role in the onset of TMJ dysfunctions. ${ }^{1}$ The present study used hydrocortisone and hyaluronic acid injections after arthrocentesis in the treatment of TMJ disorders and aimed at assessing and comparing the effectiveness of these two drugs in alleviating the symptoms.

\section{Materials And Methods}

A total of twenty patients with a chief complaint of TMJ pain, joint sounds and limited mouth opening were selected for the study and were randomly divided into 2 groups A and B. Group A was treated with arthrocentesis followed by intra-articular injections of hydrocortisoneand Group $B$ was treated with arthrocentesis followed by intra-articular injections of Hyaluronic acid. Patients who have been diagnosed with internal derangement (Anterior disc displacement with or without reduction), with moderate to severe and intractable pain in TMJ due to TMD and who have Clicking/Noises or Crepitus and who do not have any uncontrollable systemic disease were included in the study. Patients with presence of other disorders involving the TMJ (e.g., myalgia or collagen vascular disease), history of major jaw trauma, dentofacial deformity and psychiatric illness were excluded. The following parameters evaluated for the Temporomandibular J oint Pain, J oint sounds and Maximum Mouth Opening (MMO), Protrusiveand Right \& Left lateral excursions preoperatively and at 2 weeks, 1 month, 3 months and 6 months postoperatively.

\section{Methods:}

Procedure of arthrocentesis: The points of needle insertion were marked on the skin as follows (Figurel). A line was drawn from the middle of the tragus to the outer canthus (Cantho-tragal line). The posterior entrance point was located along the cantho-tragal line $10 \mathrm{~mm}$ from the middle of the tragus and $2 \mathrm{~mm}$ below the cantho-tragal line. The anterior point of entry was placed $10 \mathrm{~mm}$ farther al ong the line and $8-10 \mathrm{~mm}$ bel ow the cantho-tragal line. After the points of insertion for the two needles have been marked, two needles of 18 gauge each were selected and their position of bevel was marked on their hubs respectively (Figure 2 ). This was done as during the procedure of Arthrocentesis, the bevels of the syringes should face each other to enable the free flow of the lavage fluid. This can be ensured by making marks on their hubs. One needle was inserted into the posterior entrance point and the other one was inserted into the anterior entrance point. After insertion of the needles, it can be observed that the synovial fluid mixed with blood will come out through the needles. A $10 \mathrm{ml} / 50 \mathrm{ml}$ syringe containing normal saline solution was then connected to one of the needles (Figure 3). Proper positioning of the needle in the joint space was confirmed when injection of the solution results in its exit from the other needle.

The joint was then irrigated with $200 \mathrm{ml}$ of normal saline solution. During the lavage, the mandible was moved through opening, lateral excursive and protrusive movements to facilitate Iysis of adhesions. After the joint lavage was completed, the anterior needle was removed and then $50 \mathrm{mg}$ hydrocortisone or $1 \mathrm{ml}$ or $20 \mathrm{mg}$ hyaluronic acid was injected through the posterior needle (Figure 4 and 5).

All patients were given post-operative instructions, prophylactic antibiotics and analgesics were prescribed for 1 week. Physiotherapy was commenced immediately postoperatively to promote and maintain an improved range of mandibular movement and occlusal splint was used as supportive therapy. Patient following TMJ Arthrocentesis were assessed regularly at 2 weeks, 1 month, 3 months and 6 months postoperatively. The following parameters were recorded post operatively for both the groups: Pain on visual analogue scale, Maximum mouth opening, Protrusive movements, Left lateral excursions, Right lateral excursions and J oint sounds.

\section{RESULTS}

Out of twenty patients twelve cases were diagnosed as anterior disc displacement with reduction, eight cases were diagnosed as anterior disc displacement without reduction. The patients were between ages 19 to 46 . Out of twenty patients 9 were female patients and 11 were male patients. Pain was decreased in both the groups postoperatively at 2 weeks and $3^{\text {rd }}$ month and 


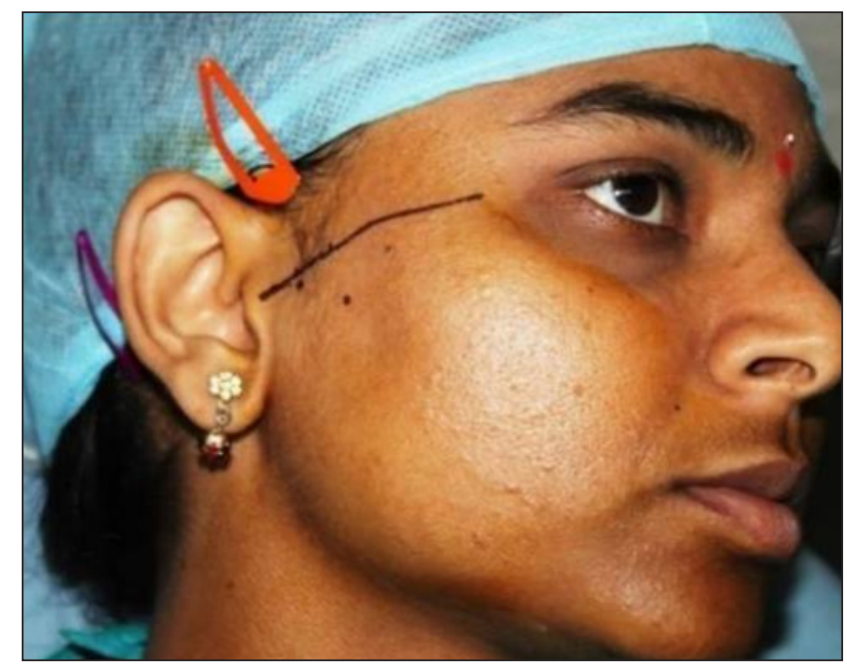

Figure 1: Marking of trago canthal line and anterior and posterior points

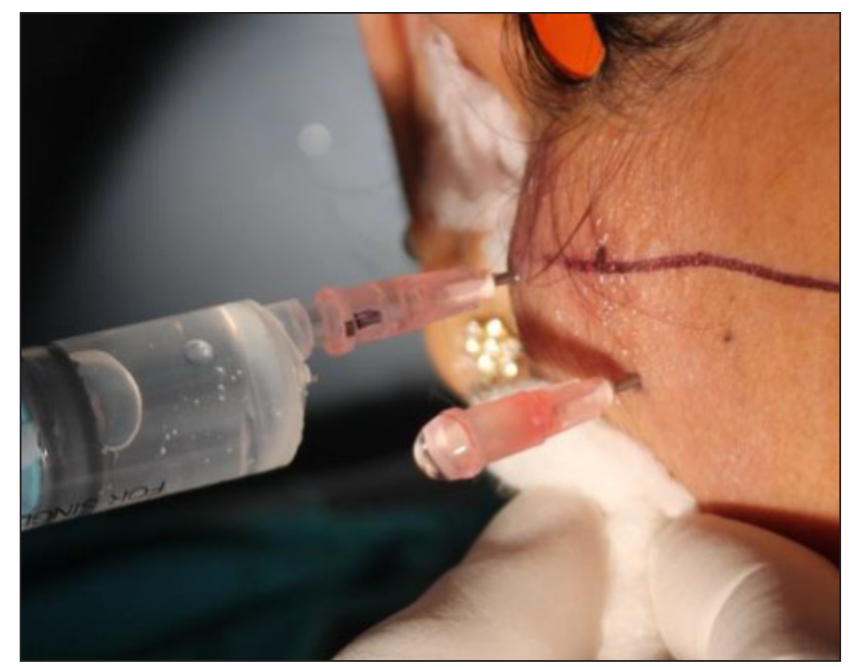

Figure 1: Figure 3: Lavage of joint with normal saline

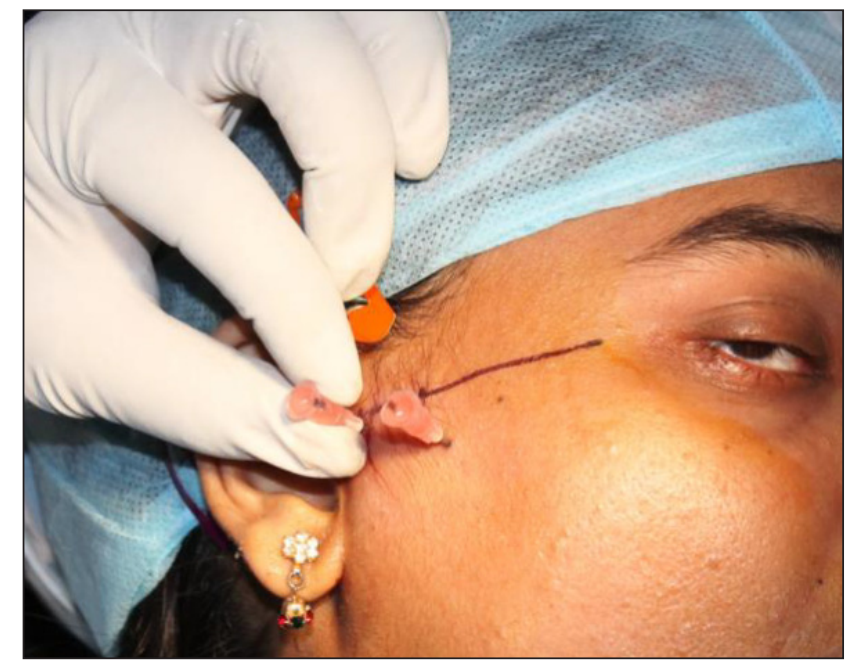

Figure 2: Placement of needles

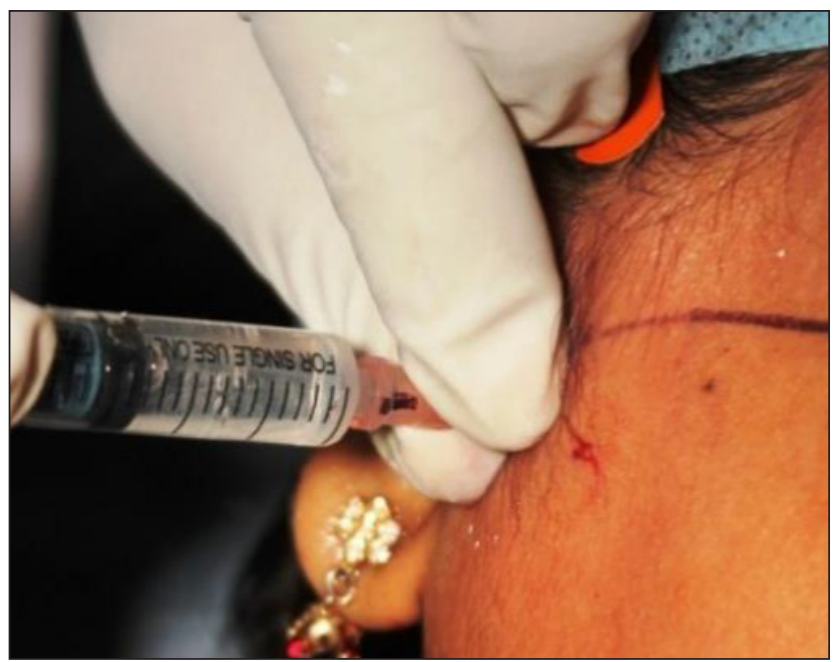

Figure 4: I njection of drug after arthrocentesis

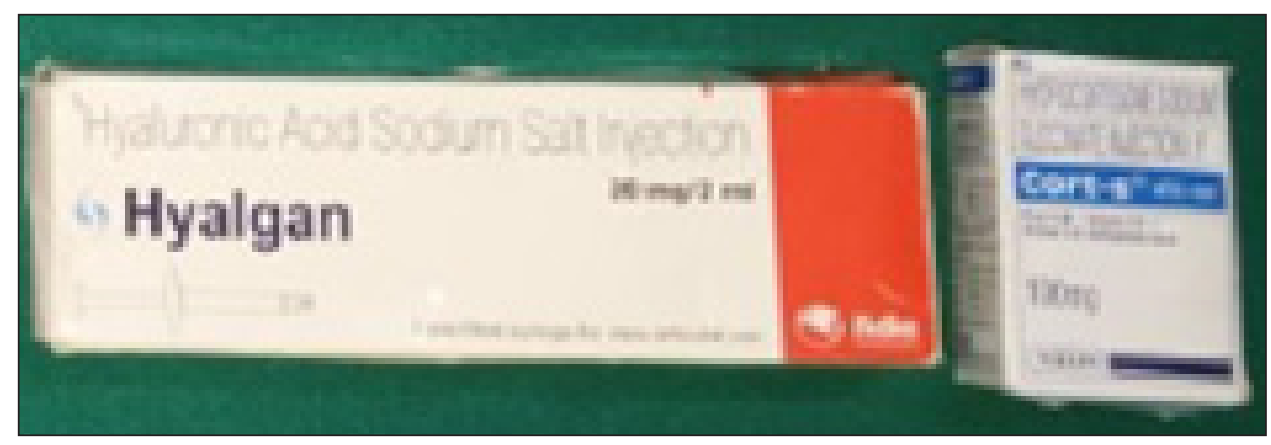

Figure 5:I njection hyaluronic acid and I njection hydrocortisone 


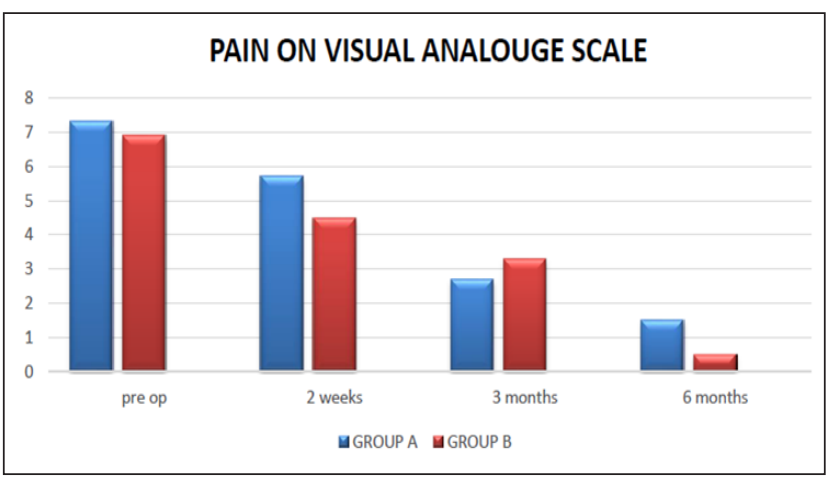

Graph 1: Pain on visual analogue scale

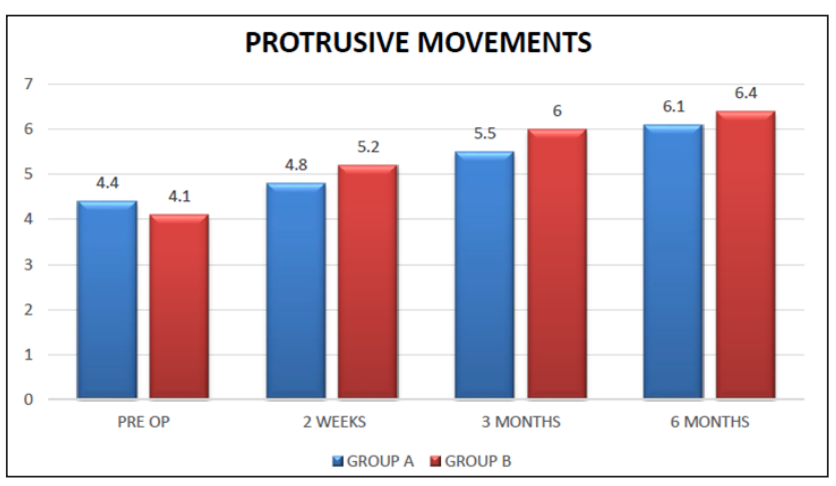

Graph 3: Protrusive movements

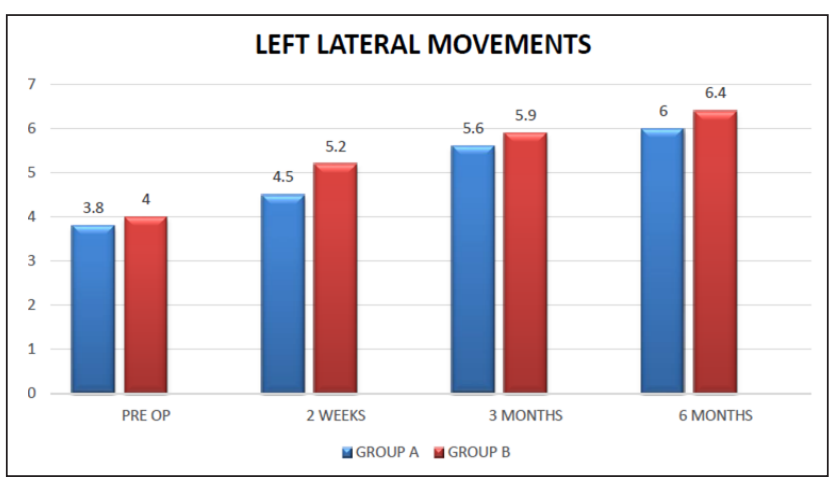

Graph 5: Left lateral movements

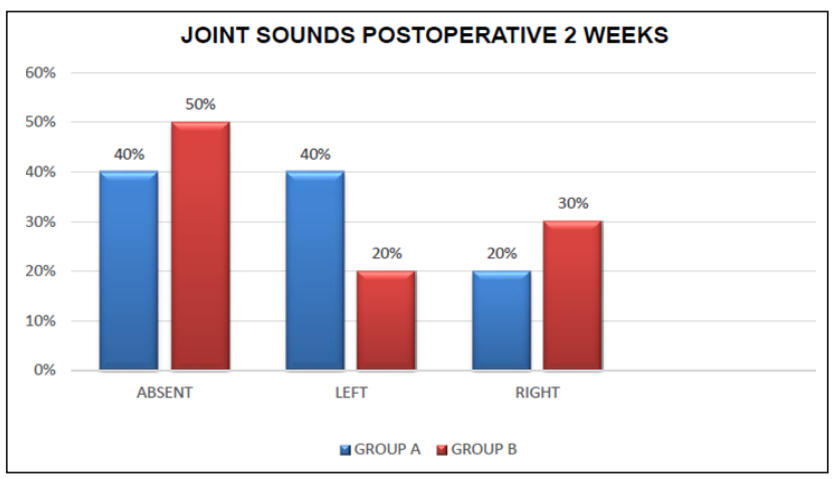

Graph 7: J oint sounds post operative 2weeks

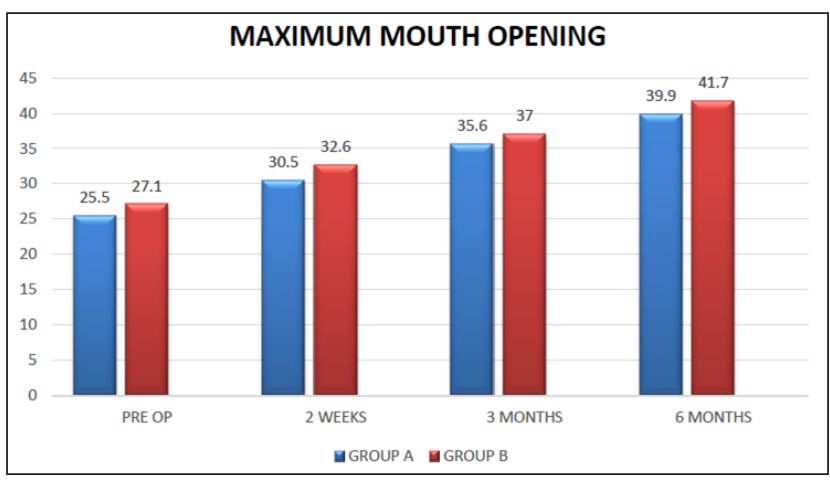

Graph 2: Maximum mouth opening

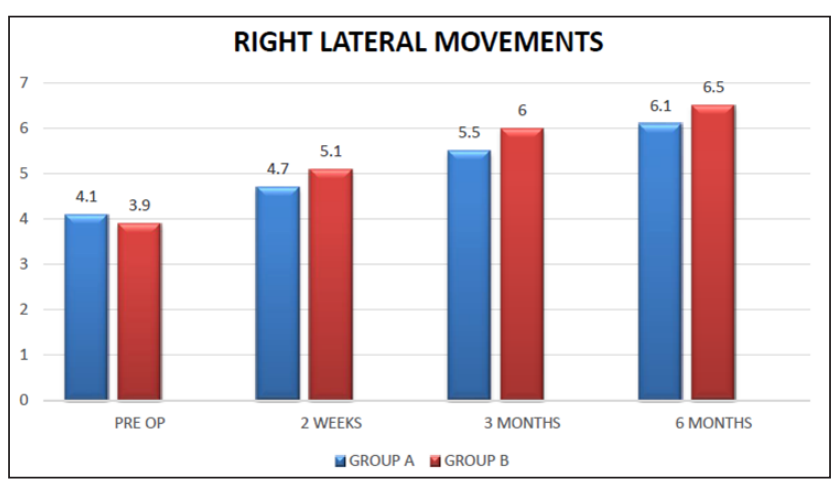

Graph 4: Right lateral movements

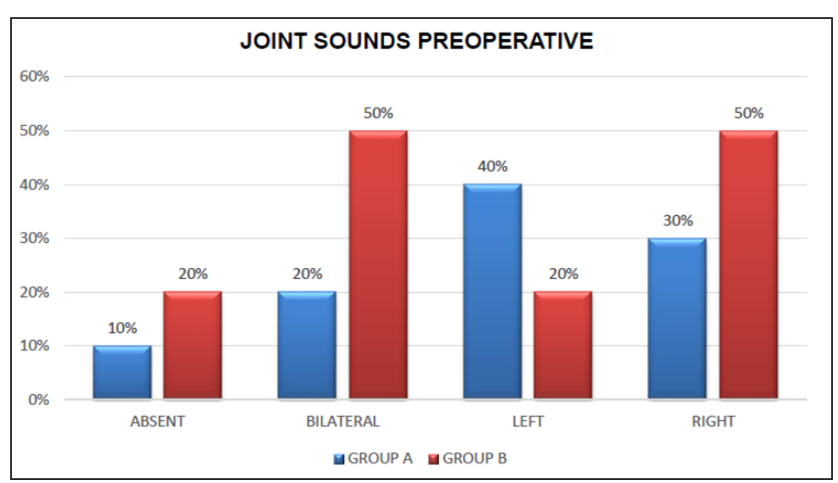

Graph 6: Preoperative J oint Sounds

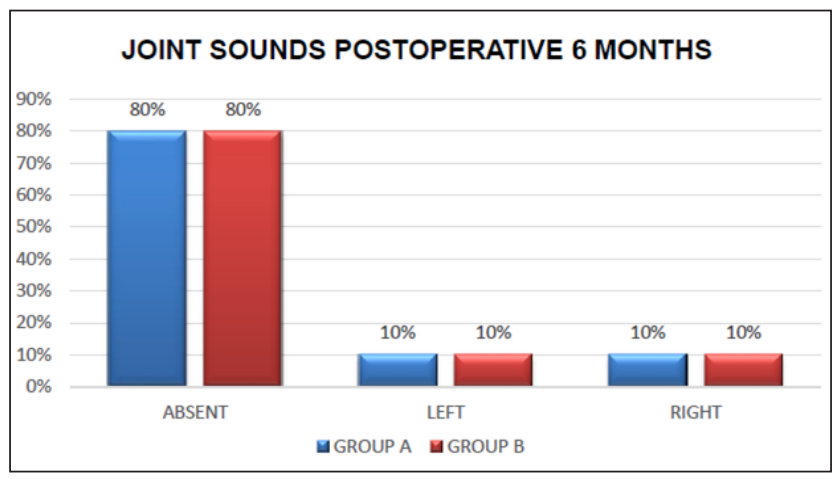

Graph 8: J oint sounds post operative 6 months 
$6^{\text {th }}$ month follow up compared to preoperative pain. According to the visual analogue scale for pain (Graph 1), intra articular injection Hyaluronic acid proved to be better compared to injection $\mathrm{Hydrocortisone}$ and there were statistically significant results between two groups. Preoperative maximum mouth opening in group $A$ patients ranged from 20 to $31 \mathrm{~mm}$ with a mean of 25.50 , while postoperative maximum mouth opening ranged from 26 to $32 \mathrm{~mm}$ with a mean of $30.50 \mathrm{~mm}$ at 2 weeks and 32 to $40 \mathrm{~mm}$ with a mean of $35.60 \mathrm{~mm}$ at 3months follow-up and 38 to 42 with a mean of 39.90 at 6 months follow up. The preoperative maximum mouth opening in Group B patients opening ranged from 23 to $31 \mathrm{~mm}$ with a mean of 27.10 , while postoperative maximum mouth opening ranged from 23 to $36 \mathrm{~mm}$ with a mean of $32.60 \mathrm{~mm}$ at 2 weeks and 34 to $40 \mathrm{~mm}$ with a mean of $37.00 \mathrm{~mm}$ at 3 months follow-up and 40 to 40 with a mean of 41.70 at 6 months follow up. The maximum mouth opening (Graph 2), protrusive movements(Graph 3), left lateral excursions (Graph 4), right lateral excursions (Graph 5) were increased in both the groups post operatively at 2 weeks and at 3rd and 6th month follow up compared to preoperative maximum mouth opening. There was no statistically significant difference between two groups. The joint sounds reduced in both the groups post operatively at 2 weeks, $3^{\text {rd }}$ and at $6^{\text {th }}$ month follow up compared to preoperative joint sounds (Graph 6, 7 and 8). There was decrease in joint sounds from $90 \%$ to $10 \%$ in group A and from $80 \%$ to $10 \%$ in group B. There was no statistically significant difference between two groups.

\section{DISCUSSION}

The Temporomandibular joint (TMJ ) is a highly adaptive organ that constantly adjusts to the functional demands made on it by means of remodeling. However, when the rate of degradation exceeds that of synthesis, the joint's modeling capacity will be insufficient to keep up with the demands, with resultant changes occurring in the joint structure. ${ }^{2}$ The TMJ disorders are characterized by a classically described triad of clinical signs: muscleand/or TMJ pain, TMJ sounds and restriction, deviation or deflection of mouth opening path.
Primary goals of the treatment for TMD are to increase the range of motion and relieve the functional pain of the TMJ . The current conservative treatments suggested for TMD include patient behavioral education, resting the jaw, soft diet, analgesic agents, splints, and physiotherapy; surgical interventions include arthrocentesis, disc repositioning, or discectomy for patients with resistant internal derangement. ${ }^{3,4}$ Recent reports have pointed out the importance of joint lubrication for a correct joint function, also hypothesizing that abnormalities of the joint lubrication system may play a role in the onset of TMJ dysfunctions. ${ }^{1}$ An efficient lubrication system in the TMJ is absolutely necessary so the disc can slide al ong the slope of the eminence. Hyaluronic acid probably plays an important indirect role in joint lubrication by adhering to surface-active phospholipids. It was found that the mechanical lysis of adhesions and lavage of the TMJ was often successful in treating various internal derangements. ${ }^{5}$ Nitzan et al first described TMJ arthrocentesis as the simplest form of surgery in the TMJ , aiming to rel ease the articular disc and to remove adhesions between the disc surface and the mandibular fossa by means of hydraulic pressure from irrigation of the upper chamber of the TMJ . ${ }^{6}$ Arthrocentesis, by definition, refers to the aspiration of fluid from a joint space and injection of a therapeutic substance. ${ }^{7,8}$ It is based on two previous treatment modalities; socalled pumping manipulation procedure to manage TMJ closed lock, and the arthroscopic lysis and lavage. Murakami et al first described a technique of TMJ arthrocentesis with pumping irrigation and hydraulic pressure to the upper joint cavity followed by manipulation of the jaw. ${ }^{9}$ Nitzan et al then described a technique whereby two needles instead of one were introduced into the upper joint space and this technique of arthrocentesis was used in the present study. ${ }^{10}$

Various pharmacological agents (I ntra-articular injections) used for alleviating TMJ pain and dysfunction after arthrocentesis. They are corticosteroids like hydrocortisone, dexamethasone, triamcinol one acetanoide, methyl prednisolone, Nonsteroidal anti-inflammatory drugs like Piroxicam, Tenoxicam, opioids like M orphine, Local anaesthetic agents like Bupivacaine, Mepivacaine and hyaluronidase, Hyaluronic acid injections. 
Intra articular injections of $50 \mathrm{mg}$ hydrocortisone and $20 \mathrm{mg}$ hyal uronic acid have been used after arthrocentesis and compared their efficacy in reducing TMD symptoms like pain, restricted mouth opening, restricted jaw movements and joint sounds.

Hyaluronic acid is a linear polysaccharides. It is available in the extracellular matrix of various mammalian tissues including skin, cartilage, umbilical cord, and synovial fluid. ${ }^{11}$ In 1939, Mayer at al first identified it in synovial fluid. Hyaluronic acid may act as a shock absorber that protects cartilage cells from shock waves, and it may also act as a barrier. ${ }^{12}$ Hyaluronic acid also has antiinflammatory actions such as scavenging for free radicals and reducing vascular permeability, as well as inhibition and phagocytosis of polymorphonuclear leucocytes and macrophages. It also has analgesic properties. Several reports haveindicated that intraarticular injection of hyaluronic acid may be effective treatment for rheumatoid arthritis, osteoarthritis, and also disorders of the TMJ .

Corticosteroids havea potent anti-inflammatory effect on synovial tissue and are known to reduce effusion, decrease pain and bring about an increase in range of motion of synovial joints. ${ }^{13}$ I ntra-articular corticosteroid injection al one or after arthrocentesis provides, long-term palliative effects on subjective symptoms and clinical signs of TMJ pain. ${ }^{14,15}$ The anti-inflammatory effects of intra-articular corticosteroids on synovial tissues have been well documented. They are useful for alleviating pain, swelling, and dysfunction in patients with inflammatory diseases of the joints such as rheumatoid arthritis and gout, as well as in those with primarily non-inflammatory joint diseases such as osteoarthritis. There are many glucocorticoid preparations such as cortisone, hydrocortisone, betamethasone, methylprednisolone acetate, triamcinolone acetonide, and triamcinolone hexacetonide.

In the immediate postoperative phase, the patient may experience some tenderness and swelling over the treated TMJ . There may also be a slight change in the bite, and on occasions, a minor hearing impairment, all of which resolve completely in a few days. Soft splint is continued and soft diet is recommended for the first few days, however active jaw opening exercises are encouraged immediately upon completion of the procedure.

No complications were encountered in our study. The complications though rare documented in the literature were infection, external auditory canal perforation, fluid extravasation into the soft tissues, bite change, scuffing of the cartilage of the TMJ , and hematoma. ${ }^{7}$ The incidence of these can be lowered by strict asepsis and using the least traumatic technique, particularly the avoidance of extravasation of fluid.

Local side effects of the intra-articular injection of glucocorticosteroids such as destruction of articular cartilage, infection, and progression of al ready recognized joint disease, have been reported. However, the cause of these deleterious effects has not been fully explained and adequate controls are lacking. In long-term treatments, serious si de effects appear, limiting the effectiveness of glucocorticoids in chronic diseases. According to our study arthrocentesis followed by injection hyaluronic acid was better than arthrocentesis followed by hydrocortisone because exogenous hyaluronic acid can stimulate the synthesis of endogenous hyaluronic acid-forming synoviocytes of osteoarthritic joints, so reducing joint friction coefficient and decreasing risk of damage and it has lesser side effects.

\section{CONCLUSION}

TMJ arthrocentesis is a simple, less invasive and less expensive technique with low morbidity and an effectiveand efficient alternativeto more invasive surgical procedures. Arthrocentesis has been reported to reduce joint pain, improve function, and reduce clicking and is ideal for early management of TMJ disorders. ${ }^{6}$ In patients who fail to respond to conventional conservative measures, in a joint that is not deemed to be grossly mechanically deranged, we advocate the use of TMJ arthrocentesis. It is observed that arthrocentesis followed by intra articular injection of hyaluronic acid was better than hydrocortisone and however additional research may require for long term evaluation of the results. 


\section{REFERENCES}

1. Guarda-Nardini L, Stifano M, Brombin C, Salmaso L, Manfredini D. A one-year case series of arthrocentesis with hyaluronic acid injections for temporomandibular joint osteoarthritis Oral Surg Oral Med Oral Pathol Oral Radiol Endod 2007; 103(6):14-22.

2. Nitzan DW, Price A. The Use of Arthrocentesis for the Treatment of Osteoarthritic Temporomandibular J oints.J Oral Maxillofac Surg 2001; 59(10):1154-1159.

3. Dimitroulis $G$, Dolwick MF, Martinez A. Temporomandibular joint arthrocentesis and lavage for the treatment of closed lock: a follow-up study. $\mathrm{Br}$ J of Oral and Maxillofac Surg 1995; 33(1):23-27.

4. Hanci M, Karamese M, Tosun Z, Aktan TM, Duman S, Savaci N. Intra-articular platelet-rich plasma injection for the treatment of temporomandibular disorders and a comparison with arthrocentesis J Craniomaxillofac Surg 2015; 43(1):162-166.

5. Nitzan DW. Arthrocentesis - Incentives for Using This Minimally Invasive Approach for Temporomandibular Disorders. Oral Maxillofac Surg Clin North Am 2006; 18(3):311-328.

6. Cavalcanti do Egito Vasconcelos B, Bessa Nogueira RV, Rocha NS. Temporomandibular joint arthrocententesis: evaluation of results and review of the literature. Braz J of Otorhinolaryngol 2006; 72(5):634-638.

7. Frost DE, Kendell BD. The Use of Arthrocentesis for Treatment of Temporomandibular J oint Disorders J Oral Maxillofac Surg1999; 57(5):583-587.

8. Yeung RW, Chow RL, Samman N, Chiu K. Short-term therapeutic outcome of intra-articular high molecular weight hyaluronic acid injection for non-reducing disc displacement of the temporomandibular joint. Oral Surg
Oral Med Oral Pathol Oral Radiol Endod 2006; 102(4): 453461.

9. Murakami KI, Matsuki M, lizuka T, Ono T. Recapturing the Persistent Anteriorly Displaced Disk by Mandibular Manipulation after Pumping and $\mathrm{Hydraulic}$ Pressure to the Upper J oint Cavity of the Temporomandibular J oint. Cranio 1987; 5(1):17-24.

10. Nitzan DW, Dolwick MF, Martinez GA. Temporomandibular J oint Arthrocentesis: A Simplified Treatment for Severe, Limited Mouth Opening J Oral Maxillofac Surg 1991; 49(11):1163-1167.

11. Sharma A, Rana AS, J ain G, Kalra P, Gupta D, Sharma S. Evaluation of efficacy of arthrocentesis (with normal saline) with or without sodium hyaluronate in treatment of internal derangement of TMJ .A prospective randomized study in 20 patients .J oral boil Craniofac Res 2013; 3(3):112-119.

12. Meyer K, Smyth EM, Dawson MH. The isolation of mucopolysaccharide from synovial fluid. J Biol Chem 1939; 128:319-327.

13. Giraddi GD, Siddaraju A, Kumar B, Singh C. Internal Derangement of Temporomandibular J oint: An Evaluation of E ffect of Corticosteroid I njection Compared with I njection of Sodium Hyaluronate after Arthrocentesis. J . Maxill ofac. Oral Surg 2012; 11(3):258-263.

14. Kopp S, Wenneberg B, Haraldson T, Carlsson GE. The Short-term Effect of Intra-articular Injections of Sodium Hyaluronate and Corticosteroid on Temporomandibular J oint Pain and Dysfunction. J Oral Maxillofac Surg 1985; 43(6):429-435.

15. Kopp S, Carlsson GE, Haraldson T, Wenneberg B. LongTerm Effect of Intra-Articular Injections of Sodium Hyaluronate and Corticosteroid on Temporomandibular J oint Arthritis. J Oral Maxillofac Surg 1987; 45(11):929935.

\section{Gain quick access to our journal online View our journal at www.nacd.in}

